\title{
ARTICLE Modified citrus pectin inhibited bladder tumor growth through downregulation of galectin-3
}

Tian Fang ${ }^{1}$, Dan-dan Liu ${ }^{2,3}$, He-ming Ning ${ }^{2,3,4}$, Dan Liu ${ }^{5}$, Jing-ya Sun ${ }^{2}$, Xiao-jing Huang ${ }^{5}$, Yu Dong ${ }^{2,6}$, Mei-yu Geng ${ }^{2,3,4}$, Shi-feng Yun ${ }^{1}$, Jun Yan $^{5,7}$ and Rui-min Huang ${ }^{2,3}$

Modified citrus pectin (MCP) is a carbohydrate enriched complex, which has been implicated in cancer treatment and prevention. However, the effects of MCP on urinary bladder cancer (UBC) are unknown. In this study, MCP was first tested in T24 and J82 human UBC cells and showed the inhibition of cell viability by the sulforhodamine $B(S R B)$ assay. The $M C P$-treated UBC cells exhibited $G_{2} / M$ phase arrest with the decrease of Cyclin B1 and phosphorylated Cdc2. Caspase-3 was also activated, leading to the cleavage of Caspase- 3 and PARP. We further explored the possible molecular mechanisms upon MCP treatment in UBC cells. Reduction of galectin-3 was observed and followed with the inactivation of Akt signaling pathway. Of note, galectin-3 knockdown by RNA interference recapitulated the MCP-mediated anti-proliferation, cell cycle arrest and apoptosis. Moreover, oral administration of MCP to the T24 xenograft-bearing nude mice inhibited the tumor growth significantly $(P<0.05)$. Quantification analysis of immunohistochemistry staining for Ki67 and cleaved Caspase- 3 confirmed the decrease of proliferation index $(P<0.05)$ and the increase of apoptosis index $(P<0.01)$ in $700 \mathrm{mg} / \mathrm{kg}$ MCP-fed UBC xenografts. Using the information from TCGA database, we revealed that the overexpression of galectin-3 was associated with high tumor grade with lymph node metastasis, poor overall survival in UBC patients. Considering the remarkable inhibitory effects of MCP on UBC cell proliferation and survival in vitro and in vivo mainly through galectin-3, which is upregulated in UBCs, MCP may become an attractive agent, as a natural dietary fiber, for prevention and therapy of UBCs.

Keywords: modified citrus pectin; urinary bladder cancer; galectin-3; xenograft; cell survival

Acta Pharmacologica Sinica (2018) 39:1885-1893; https://doi.org/10.1038/s41401-018-0004-z

\section{INTRODUCTION}

Urinary bladder cancer (UBC) is the 6th most common cancer in men world-wide in 2012 [1]. It is also the 4th most common cancer in men in the USA with an estimated 60,490 new cases in 2017 [2]. Although surgical resection of the bladder can be curative, half of cystectomy patients still recur with metastases within two years [3]. Moreover, frequent follow-up and monitoring, as well as repeated clinical interventions after surgery, lead to lifelong management, which makes UBC far costlier than any other cancers [4].

Modified citrus pectin (MCP) is derived from the peel and pulp of citrus fruits and modified into a complex water soluble indigestible polysaccharide processed by high temperature and high pH [5]. Clinical importance of dietary components (including $\mathrm{MCP})$ has been demonstrated in cancer prevention and treatment. Recent studies including our previous data supported that MCP possesses anti-tumor properties against melanoma and prostate cancer [6-8]. The main target of MCP is galectin-3 [9]. whose overexpression has been implicated in multiple cancer types, such as breast, lung, gastric and liver cancers [10]. As a unique chimeric member of the $\beta$-galactoside-binding protein family, galectin- 3 is a multivalent and multifunctional oncogenic protein involved in cell growth, cell differentiation, cell adhesion, RNA splicing, cell apoptosis, malignant transformation and metastasis [11,12]. Notably, galectin-3 also acts as a guardian of the tumor microenvironment to suppress immune surveillance by killing $\mathrm{T}$ cells and interfering with NK cell function [13]. Possible therapeutic strategies using some carbohydrate ligands such as MCP to block galectin-3 activity for cancer therapy are currently investigated [10].

The elevated expression of galectin-3 has also been reported in UBC samples [14,15]. Thus, we explore whether targeting galectin3 by dietary MCP is a therapeutic option for bladder cancer treatment in this study. The effects of MCP on cell survival, cell cycle distribution, and apoptosis were tested in two human UBC cell lines, T24 and J82. Suppression of galectin-3-mediated Akt activity by MCP was demonstrated in UBC cells. MCP markedly inhibited tumor growth of T24 xenografts in vivo as well. These data suggest that targeting galectin-3 by dietary MCP has a great

\footnotetext{
${ }^{1}$ Department of Comparative Medicine, Jinling Hospital, Clinical School of Medical College of Nanjing University, Nanjing 210002, China; ${ }^{2}$ Shanghai Institute of Materia Medica, Chinese Academy of Sciences, Shanghai 201203, China; ${ }^{3}$ University of Chinese Academy of Sciences, Beijing 100049, China; ${ }^{4}$ Shanghai Tech University, Shanghai 201210, China; ${ }^{5}$ State Key Laboratory of Pharmaceutical Biotechnology and MOE Key Laboratory of Model Animals for Disease Study, Model Animal Research Center of Nanjing University, Nanjing 210061, China; ${ }^{6}$ Shanghai University, Shanghai 200444, China and ${ }^{7}$ Collaborative Innovation Center of Genetics and Development, Shanghai 200438, China Correspondence: Shi-feng Yun (yunshifeng1@163.com) or Jun Yan (yanjun@nju.edu.cn) or Rui-min Huang (rmhuang@simm.ac.cn) These authors contributed equally: Tian FANG, Dan-dan LIU, and He-ming NING.
}

Received: 25 September 2017 Accepted: 8 January 2018

Published online: 16 May 2018 
potential to treat UBC, which might be a subject for clinical practice in the future.

\section{MATERIALS AND METHODS}

Cell culture

Human UBC cell lines, T24 and J82, were obtained from the Cell Bank of Type Culture Collection, Chinese Academy of Science (Shanghai, China). Both cell lines were maintained in RPMI 1640 medium (Hyclone, Logan, UT), supplemented with $10 \%$ fetal bovine serum (Hyclone). Cells were incubated at $37{ }^{\circ} \mathrm{C}$ in an atmosphere of $5 \mathrm{CO}_{2}$ and $95 \%$ air.

Cell proliferation assay

Cells were seeded into 96-well plates at a density of 3000-5000 cells/well in triplicate. PectaSol-C MCP (EcoNugenics, Santa Rosa, CA, USA) was dissolved in the cell culture medium (ranging from 0.125 to $2 \%, w / v)$. One day later, the cells were treated using MCP with different concentrations for another $72 \mathrm{~h}$. The cells were then fixed with $10 \%$ trichloroacetic acid over $4 \mathrm{~h}$ and stained with 4 $\mathrm{mg} / \mathrm{ml}$ sulforhodamine B (SRB; Sigma-Aldrich, St Louis, MO, USA) in $1 \%$ acetic acid. The protein-bound dye is dissolved in $10 \mathrm{mM}$ Tris base solution for OD determination at $560 \mathrm{~nm}$ using a microplate reader (SpectraMAX 190; Molecular Devices, Sunnyvale, (A, USA).
Cell cycle analysis

Cells were seeded into 12 -well plates at a density of $1 \times 10^{5}$ cells/ well in triplicate. $24 \mathrm{~h}$ later, the cells were treated with MCP for another $24 \mathrm{~h}$ at $37^{\circ} \mathrm{C}$. Then the adherent cells were collected and fixed in cold $70 \%$ ethanol at $4{ }^{\circ} \mathrm{C}$ for $4 \mathrm{~h}$. Cells were washed with cold PBS twice and stained with the solution containing $100 \mathrm{mg} /$ $\mathrm{ml}$ propidium iodide (Sigma-Aldrich) and $20 \mathrm{mg} / \mathrm{ml}$ RNase A (Sigma-Aldrich) at $37{ }^{\circ} \mathrm{C}$ for $30 \mathrm{~min}$ in the dark. FACS analysis were performed using a BD FACSCalibur flow cytometer (BD Biosciences, San Jose, CA, USA) and cell cycle distribution was quantified with FlowJo software (version 10.0.7; Ashland, OR, USA).

\section{Western blotting assay}

Total protein from MCP-treated cells was isolated with the RIPA buffer (Beyotime) and quantified using a Bradford protein assay kit (Bio-Rad, Hercules, CA, USA). 10-20 $\mu \mathrm{g}$ protein lysates were separated by SDS-PAGE and transferred to nitrocellulose membranes, probed with the primary antibodies followed by the corresponding horseradish peroxidase (HRP)-conjugated secondary antibodies. HRP activity on immunoblots was detected by an ECL plus detection reagent (Pierce, Rockford, IL, USA). Antibodies against Cdc2, phospho-Cdc2 (Tyr15), PARP, cleaved PARP (Asp214), Caspase-3, Akt (pan), phospho-Akt (Ser473), Bad, phospho-Bad (Ser136), S6 ribosomal protein and phospho-S6 ribosomal protein (Ser240/244), Erk1/2, and phospho-Erk1/2 a

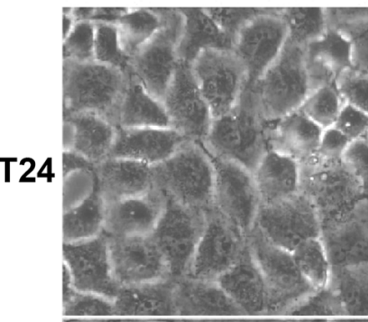

J82

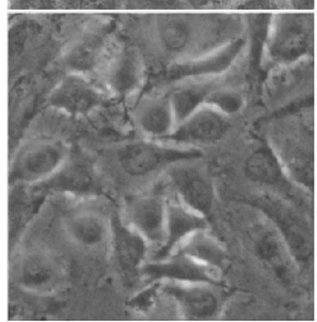

b

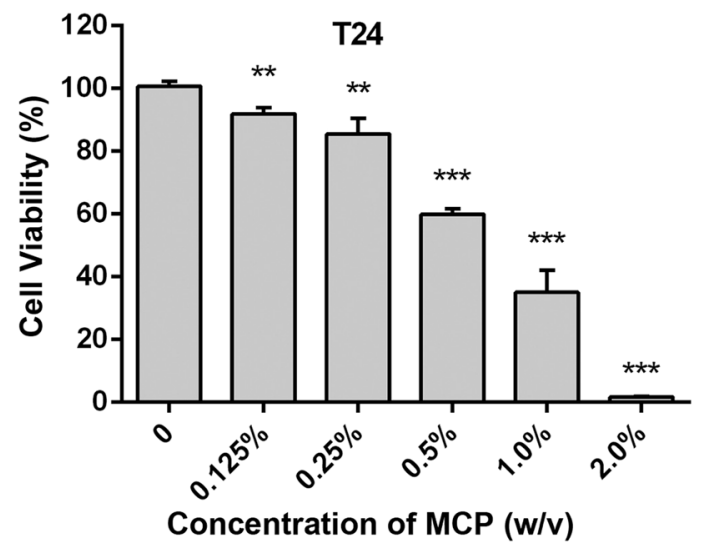

$1.0 \%$

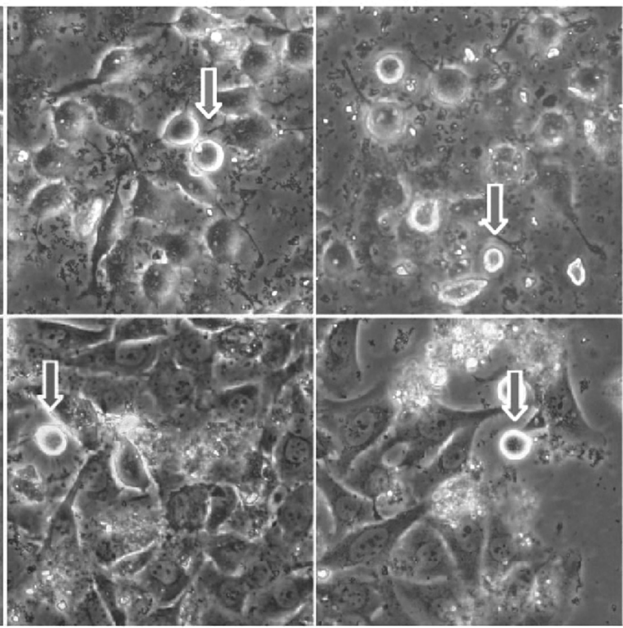

$2.0 \%$

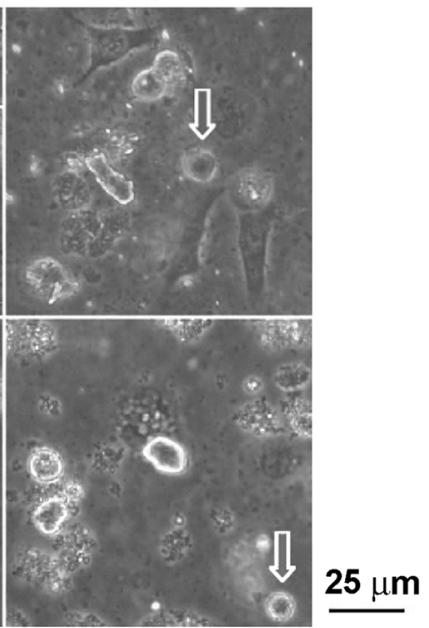

C

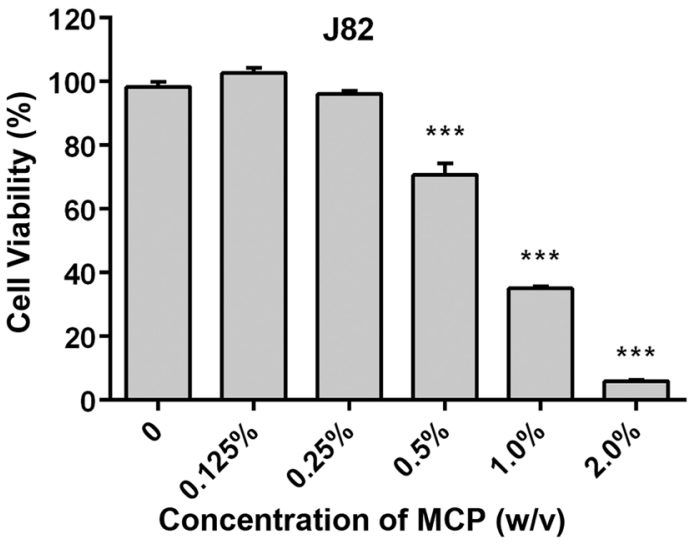

Fig. 1 MCP impaired the viability of UBC cells. a Morphological changes of T24 and J82 UBC cells upon MCP treatment for $48 \mathrm{~h}$. $\mathbf{b}$ Effects of MCP on cell viability by the SRB assay. T24 and J82 UBC were treated with MCP at the indicated concentrations for $72 \mathrm{~h}$. All experiments were independently repeated three times. Bars represented mean \pm SD vs. vehicle control. ${ }^{* *} P<0.01 ;{ }^{* * *} P<0.001$ 
a

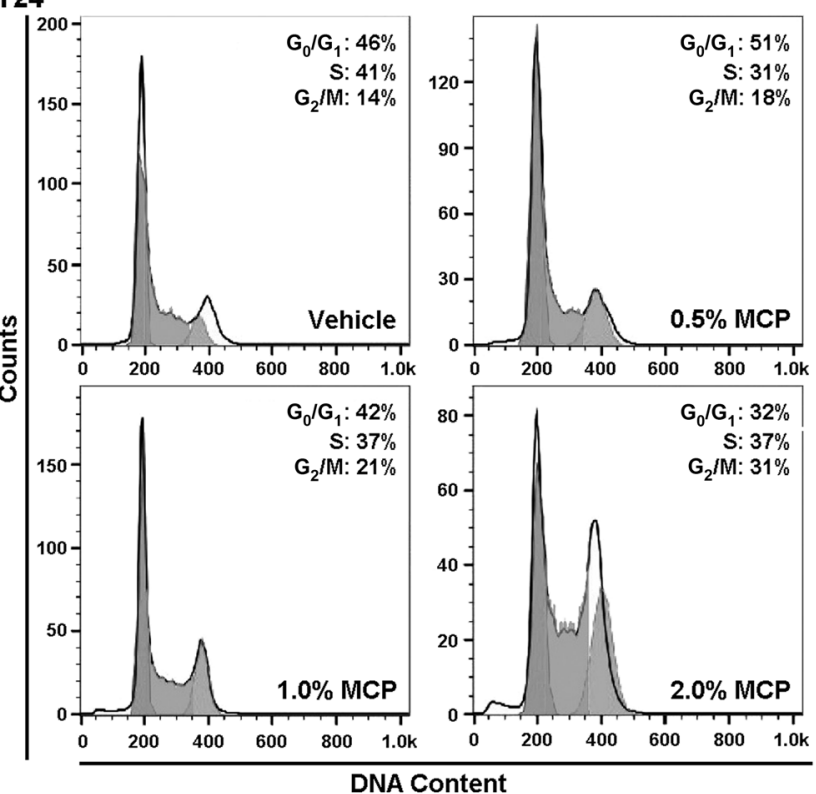

C J82

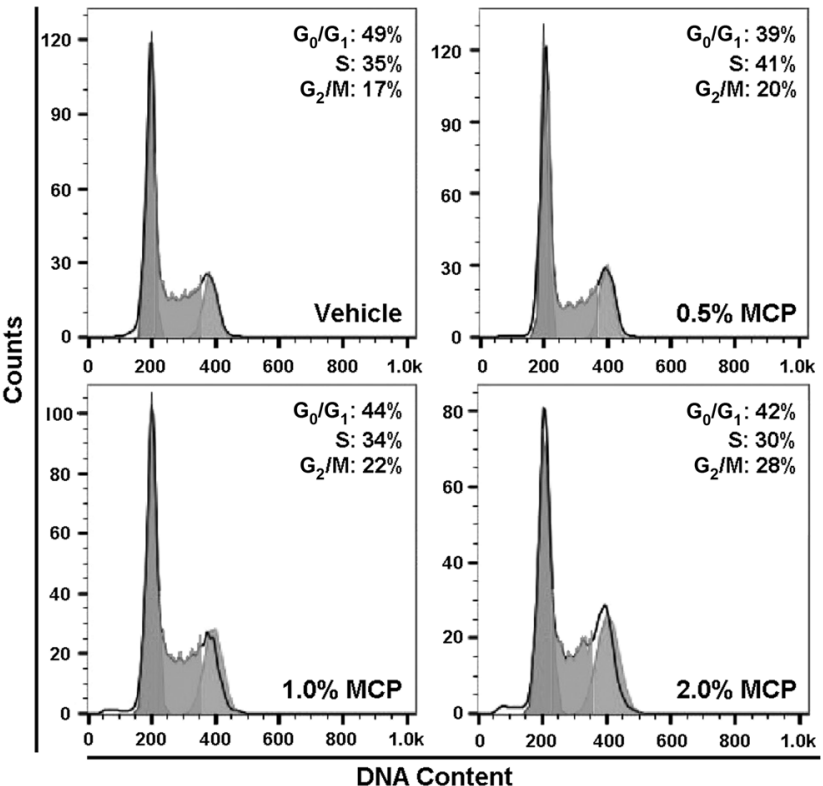

b

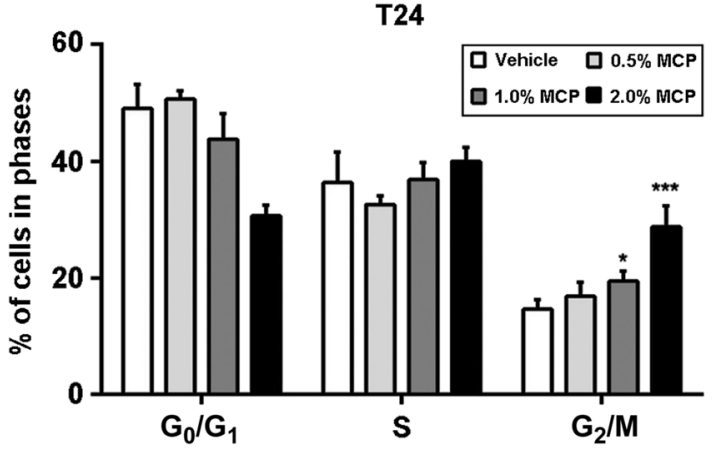

d

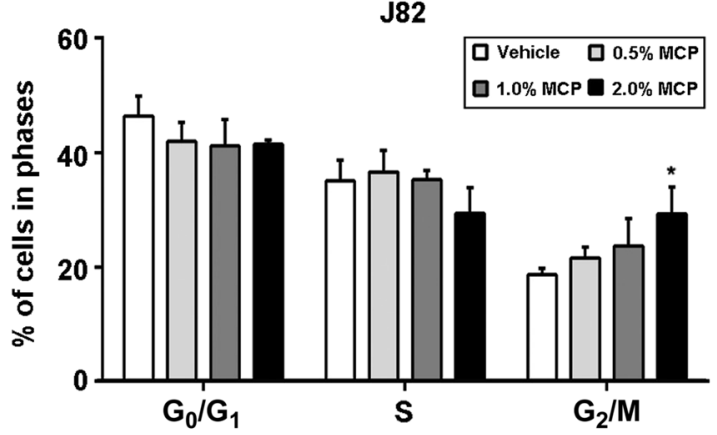

T24

\begin{tabular}{|c|c|c|}
\hline 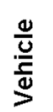 & 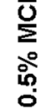 & 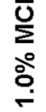 \\
\hline
\end{tabular}

Cyclin B1

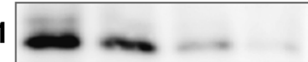

p-Cdc2

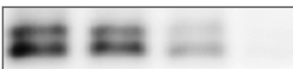

Cdc2
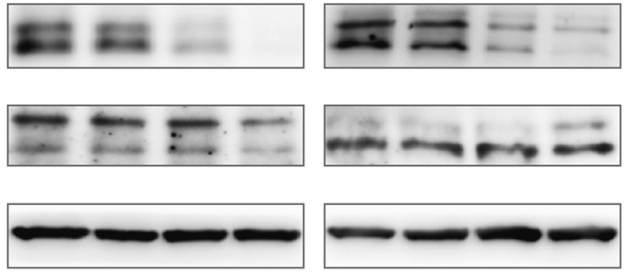

Fig. 2 MCP induced $\mathrm{G}_{2} / \mathrm{M}$ cell cycle arrest in UBC cells. Effects of MCP on cell cycle distribution of T24 cells (a, b) and J82 cells (c, d). The cell cycle distribution was analyzed by FACS after propidium iodide staining and a representative experiment was shown (a, c). Quantification results from three independent experiments were presented (b, d). Bars represented mean $\pm \mathrm{SD}$. ${ }^{*} P<0.05 ;{ }^{* * *} P<0.001$. (e) Effects of MCP on $\mathrm{G}_{2} / \mathrm{M}$ transition regulators by Western blotting assay

(Thr202/Tyr204) were purchased from Cell Signaling Technologies (Danvers, MA, USA). Cyclin B1, galectin-3 and $\beta$-Actin antibodies were obtained from Santa Cruz Biotechnology (Dallas, TX, USA), Abcam (Cambridge, MA, USA) and Sigma-Aldrich, respectively.

\section{Apoptosis analysis}

Apoptosis was assessed using the Caspase-3 assay kit (Beyotime, Shanghai, China), according to the manufacturer's instruction. Briefly, $50 \mu$ total protein lysate from MCP-treated cells was added into $50 \mu \mathrm{l}$ reaction buffer containing $10 \mu \mathrm{l}$ Caspase- 3 substrate (AcDEVD-pNA; $2 \mathrm{mM}$ ). The mixture was incubated at $37^{\circ} \mathrm{C}$ for $2 \mathrm{~h}$ and $\mathrm{OD}$ at $405 \mathrm{~nm}$ was determined with a microplate reader (Molecular Devices).
Hoechst 33342 staining

T24 cells were seeded into cell culture dishes with glass bottom (Nest Scientific, Wuxi, China) at a density of $1 \times 10^{5} /$ dish. $12 \mathrm{~h}$ later, the cells were treated with $2 \%$ MCP $(\mathrm{w} / \mathrm{v})$ for another $36 \mathrm{~h}$ at $37^{\circ} \mathrm{C}$. The cells were then washed with PBS, and stained with Hoechst $33342\left(5 \mu \mathrm{g} / \mathrm{ml}\right.$; Invitrogen, Carlsbad, CA, USA) for $5 \mathrm{~min}$ at $25^{\circ} \mathrm{C}$. The cell images were acquired by a Leica TCS SP8 confocal microscope (Wetzlar, Germany).

Galectin-3 expression in human UBC samples from public database

TCGA portal was used to obtain galetin-3 mRNA expression data (via RNASeq V2 RSEM) for UBC studies. In the dataset (TCGA Nature 2014) [16]. normalized mRNA expression levels of galetin-3 

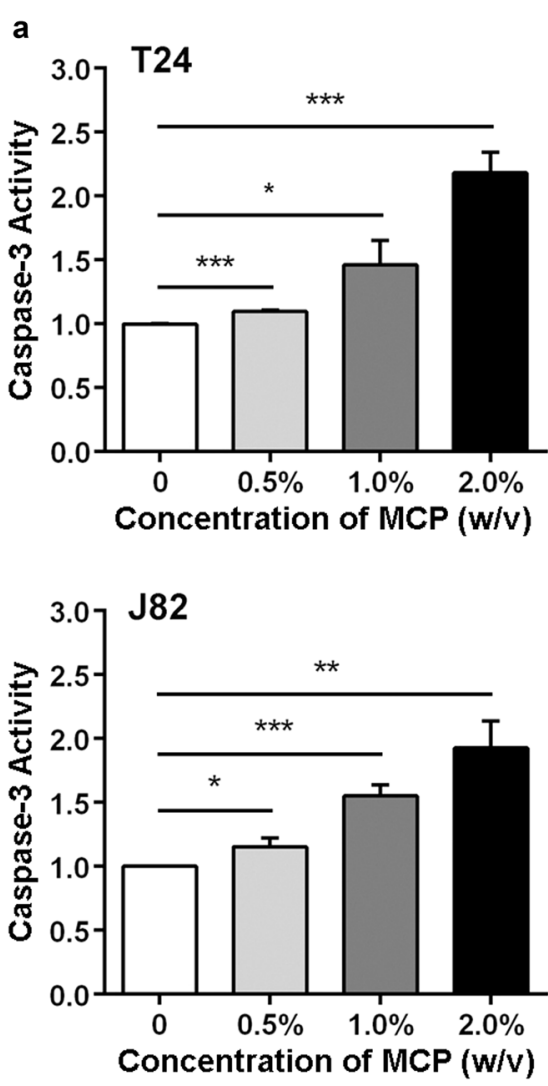

b

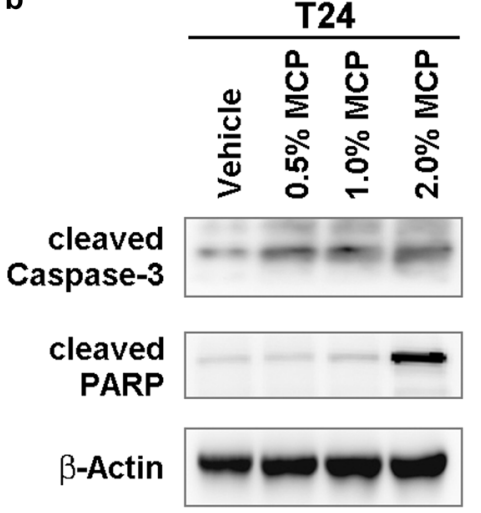

C

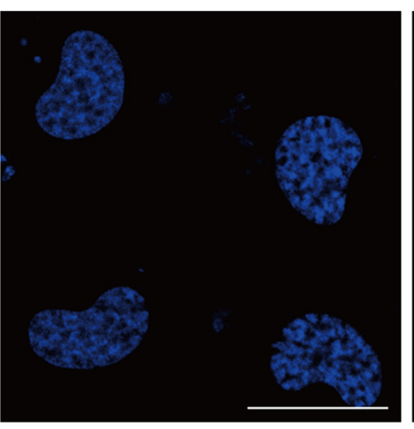

$\mathrm{J82}$
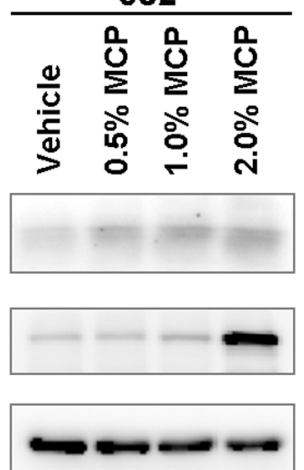

$2.0 \% \mathrm{MCP}$

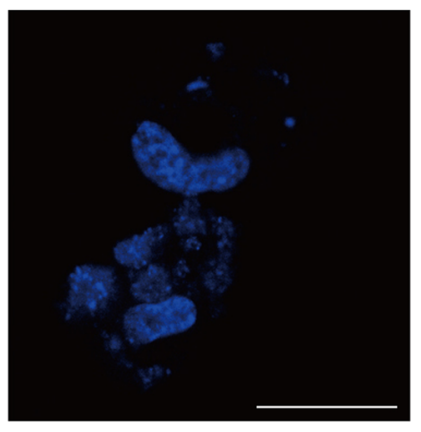

Fig. 3 MCP induced apoptosis in UBC cells. a Caspase-3 activities in T24 and J82 cells treated with MCP at indicated concentrations for $48 \mathrm{~h}$. Bars represented mean \pm SD vs. vehicle control. ${ }^{*} P<0.05 ;{ }^{* *} P<0.01 ;{ }^{* *} P<0.001$. b Effects of MCP on apoptotic proteins by Western blotting assay. c Representative images showed morphological changes of MCP-treated T24 cells using Hoechst 33342 staining. Scale bars, $25 \mu \mathrm{m}$

were analyzed in patients with lymph node metastasis $(\geq \mathrm{N} 1, n=$ 43) and without lymph node metastasis (NO, $n=73$ ).

Correlation between galectin-3 mRNA level and cumulative overall survival (OS) in human UBC samples was analyzed by the Kaplan-Meier method in two public datasets, TCGA Nature 2014 [16]. $(n=129)$ and TCGA Provisional $(n=405$, http://www. cbioportal.org/study?id=blca_tcga\#summary), respectively. Lower quartile was determined as the cut-point for galectin-3 expression and the differences in the survival rates between curves were assessed by the log-rank test. $P<0.05$ was considered statistically significant.

\section{siRNA transfection}

T24 cells were seeded in 6-well plates at $3 \times 10^{4}$ cells/well and were transfected with siRNAs using Lipofectamine 2000 (Invitrogen) according to the manufacturer's protocol. Two siRNAs targeting different regions of galectin-3 gene and control siRNA were synthesized by Genpharma (Shanghai, China) as below: siGal3-1: $5^{\prime}$ -GCU CAC UUG UUG CAG UAC ATT-3'; siGal3-2: 5'-CCA CGC UUC AAU GAG AAC ATT-3'; siNC: 5'-UUC UCC GAA CGU GUC ACG UTT-3'.

Animal studies

The animal protocol was approved by the Administrative Panel on Laboratory Animal Care of Clinical College of Nanjing University. In brief, $3.0 \times 10^{6}$ T24 cells in $0.1 \mathrm{ml} 50 \%$ Matrigel (BD Biosciences) were injected subcutaneously into the right flank of 6-week-old male athymic nude mice (PLA Academy of Military Science, Beijing, China). Two weeks after cell inoculation, the animals with palpable tumors were randomly divided into three experimental groups: (1) high-dose MCP group: $700 \mathrm{mg} / \mathrm{kg}$ body weight, $n=4$; (2) low-dose MCP group: $350 \mathrm{mg} / \mathrm{kg}$ body weight, $n=4$; 3) Vehicle group: $n=4$. MCP was dissolved in distilled water and administered orally using mouse gastric lavage (Gauge \#12) daily for 6 weeks. Tumor size was measure by digital caliper and tumor volume was calculated by the formula: tumor volume $\left[\mathrm{mm}^{3}\right]=$ (length $[\mathrm{mm}]) \times(\text { width }[\mathrm{mm}])^{2} / 2$. At the endpoint, tumors were weighted and embedded in paraffin for further histological analysis.

Immunohistochemistry (IHC)

Five- $\mu$ m-thick paraffin sections were processed and stained with primary antibodies against Ki67 (1:10000, Vector Laboratories, Burlingame, CA), cleaved Caspase-3 (1:500, Cell Signaling Technologies), and galectin-3 (1:100, Abcam), followed by the detection using UltraSensitive SP IHC kit (MXB Biotechnologies, Fuzhou, China). A rabbit IgG (1:100; Vector Laboratories) was used as the negative control for immunohistochemical staining to evaluate non-specific binding of Ki-67, cleaved caspase- 3 , and galectin-3 antibodies since all of them were made in rabbit.

Statistical analysis

Values were presented as the mean $\pm S D$, and significance between two sets of data was determined by Student's $t$-test. Differences were considered statistically significant for $P<0.05$. All statistical analyses were performed using GraphPad Prism software. 
T24

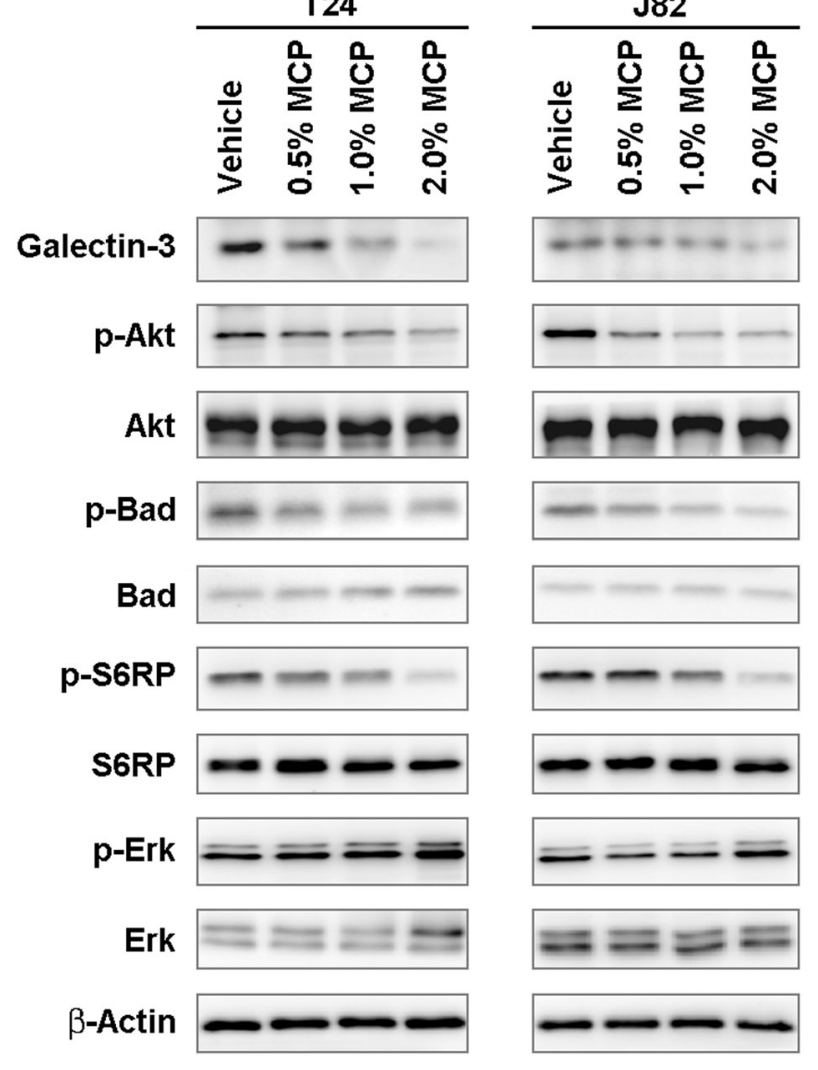

Fig. 4 MCP suppressed Galectin 3 expression and Akt signaling pathway. T24 and J82 cells were treated with MCP with the indicated concentrations for $48 \mathrm{~h}$, followed by Western blotting assay

\section{RESULTS}

The cytotoxic effect of MCP on UBC cells in vitro

Two widely used human UBC cell lines, T24 and J82, were used to examine the cytotoxic effect of MCP. Different concentrations of MCP ranging from $0.125 \%$ to $2.0 \%(\mathrm{w} / \mathrm{v})$ were applied to these cells for $72 \mathrm{~h}$. UBC cells became swollen and detached from the culture plates when MCP concentration was $\geq 0.5 \%$ (Fig. 1a). Consistently, MCP showed a significant cytotoxic effect on both UBC cell lines $(P<0.05)$ in a concentration-dependent manner (Fig. 1b).

\section{MCP induces $G_{2} / M$ phase arrest}

To determine whether MCP affects UBC cell growth on cell cycle progression, UBC cells treated with MCP for $36 \mathrm{~h}$ were harvested and the cell cycle distribution was assessed by propidium iodide staining-based flow cytometry. Apparent $\mathrm{G}_{2} / \mathrm{M}$ phase arrest was observed in both T24 and J82 cells with MCP treatment (Fig. 2a-d). For example, $28.7 \pm 3.6 \%$ of $2.0 \%$ MCP-treated T24 cells were arrested in $G_{2} / M$ phase, comparing with that $14.6 \pm 1.6 \%$ of vehicle-treated T24 cells were remained in $G_{2} / M$ phase $(P<0.05)$. Western blotting analysis revealed that the protein expression levels of Cyclin B1 and phosphorylated-Cdc2, which are key regulators for $G_{2} / M$ transition, were reduced in a concentrationdependent manner (Fig. 2e). However, the basal expression of Cdc2 did not show strong change even in 2\% MCP-treated UBC cells. These data suggested that $M C P$ can induce $G_{2} / M$ arrest in UBC cells through downregulation of Cyclin B1 and phosphorylation of $\mathrm{Cdc} 2$.

MCP induces the caspase- 3 dependent apoptosis In order to examine whether MCP can induce apoptosis in UBC cells, the Caspase-3 activities in MCP-treated cells were assessed based on its capability to cleave the substrate with the sequence DEVD by spectrophotometric detection of the chromophore $p$ nitroaniline (pNA) after cleavage from the peptide substrate DEVD-pNA. Comparison of the absorbance of pNA from $2 \%$ MCPtreated UBC cells and vehicle-treated control cells shows at least 2folds increase in Caspase-3 activity (Fig. 3a). The cleavages of Caspase- 3 and PARP, which are cellular apoptotic markers, were observed in both of T24 and $\mathrm{J} 82$ cells after incubation with $2 \%$ MCP for $48 \mathrm{~h}$ (Fig. 3b). Furthermore, numerous MCP-treated T24 cells exhibited the fragmented nuclei (Fig. 3c), the main characteristic of apoptosis.

MCP inhibits galectin-3 expression and suppresses Akt signaling To investigate how MCP exerts its inhibitory effects on UBC cells, the potential target [17-19, and downstream signaling pathways [20] of MCP were analyzed by Western blotting assays. As shown in Fig. 4, MCP decreased the expression level of its target, Galectin3 protein. We also found that phosphorylation level of Akt was reduced remarkably under the treatment of MCP. Consistently, we observed the reduced levels of $\mathrm{p}$-Bad, phosphorylated at Ser136 by $\mathrm{p}$-Akt to promote cell survival, and $\mathrm{p}$-S6 ribosomal protein ( $\mathrm{p}$ S6RP), phosphorylated at Ser240/244 by p-Akt to facilitate protein synthesis in cell cycle progression. However, MAPK signaling did not show significant change, as evidenced by p-Erk1/2 (Thr202/ Tyr204) level. These data indicate that MCP may suppress Akt signaling pathway via Galectin-3 in UBC cells.

Galectin-3 is required for UBC cell cycle arrest and apoptosis To test whether galectin-3 is implicated in UBC development, expression level of galectin-3 in UBC patients was analyzed using the public TCGA database. In the dataset (TCGA Nature 2014) [16] containing 116 UBC samples, normalized mRNA expression levels of galetin-3 was overexpressed in UBC patients with lymph node metastasis ( $\geq \mathrm{N} 1, n=43$ ) compared with those without lymph node metastasis (N0, $n=73 ; P=0.032$, Fig. 5a). Correlation between galectin-3 mRNA level and cumulative overall survival (OS) in human UBC samples was further analyzed in two datasets, TCGA Nature $2014(n=129)$ and TCGA Provisional $(n=405)$, respectively. The UBC patients were divided into two groups, with low and high expression of galectin-3, using the lower quartile as the cutoff point. The Kaplan-Meier survival analysis showed that galectin-3 expression level was inversely associated with OS $(P<$ 0.05 ) (Fig. 5b). It indicates that overexpression of galectin-3 is related to high tumor grade, poor survival and a potential therapeutic target in human UBC patients.

The role of galectin-3 in UBC cells was studied with galectin-3 gene knockdown using a RNA interfering approach in T24 cells. Two siRNAs targeting different regions of galectin-3 mRNA were tested and downregulation of galectin-3 gene expression was confirmed by Western blotting (Fig. 5c). Downregulation of galectin-3 reduced cell viability by SRB assay (Fig. $5 \mathrm{~d}$ ) and induced $\mathrm{G}_{2} / \mathrm{M}$ phase arrest (Fig. 5e, f), with the decrease of Cyclin B1 and phosphorylated-Cdc2 (Fig. $5 \mathrm{~g}$ ). Moreover, galectin-3 gene knockdown activated Caspase-3 (Fig. 5h) and increased the accumulation of cleaved Caspase-3 and cleaved PARP (Fig. 5i). Introduction of galectin-3 siRNAs into UBC cells reduced the phosphorylation of Akt, as well as its downstream target Bad (Fig. 5i). Taken together, inhibition of galectin-3 gene expression resulted in cell cycle arrest and apoptosis, consistent with the effects induced by MCP treatment.

Cell viability in galectin-3 knockdown cells upon MCP treatment was further tested. Our result showed siGal-3 treatment could partially rescue the MCP-induced cell growth inhibition (Fig. 5j; $P$ $<0.01$ ), which confirms the inhibitory effect of MCP on bladder cancer cells is galectin-3 dependent. However, galectin-3 knockdown could not completely reverse the MCP-induced inhibitory effect, indicating that galectin-3 may be one of MCP's targets. 


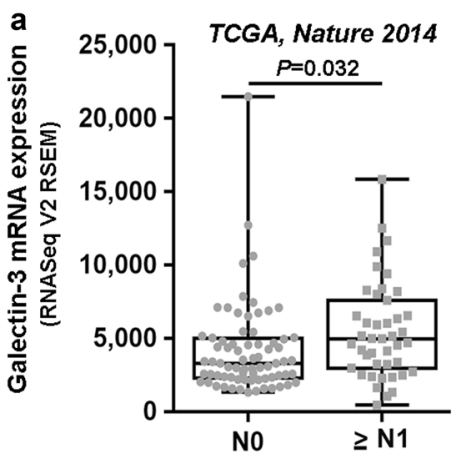

b

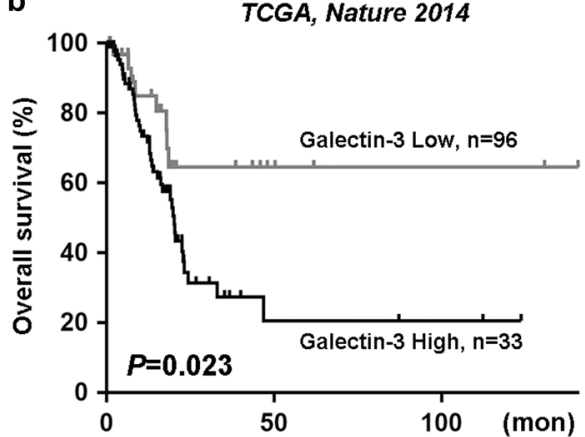

d

C

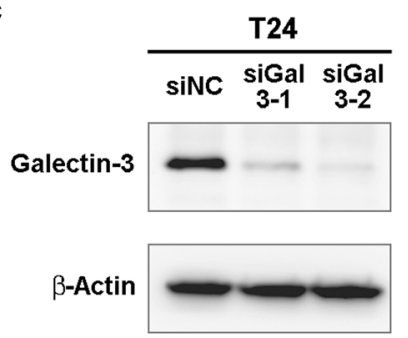

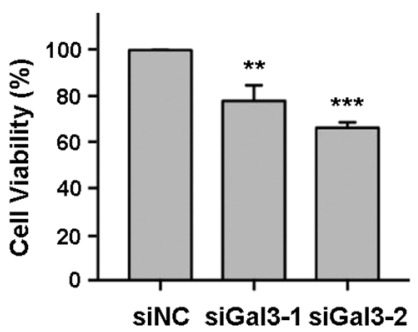

f

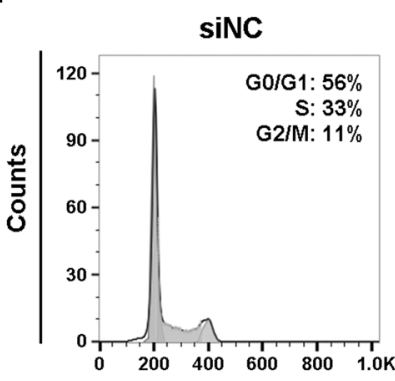

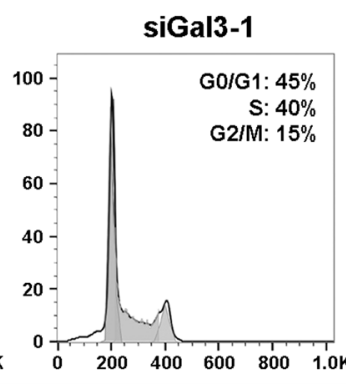

DNA Content

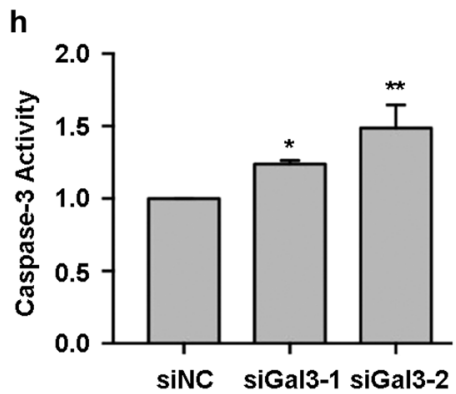

i

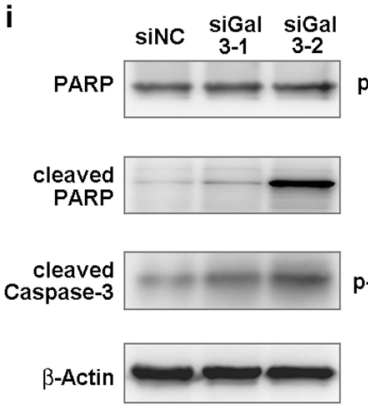

siGal3-2

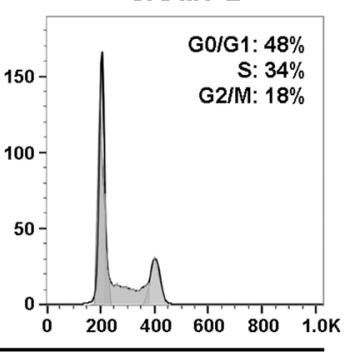

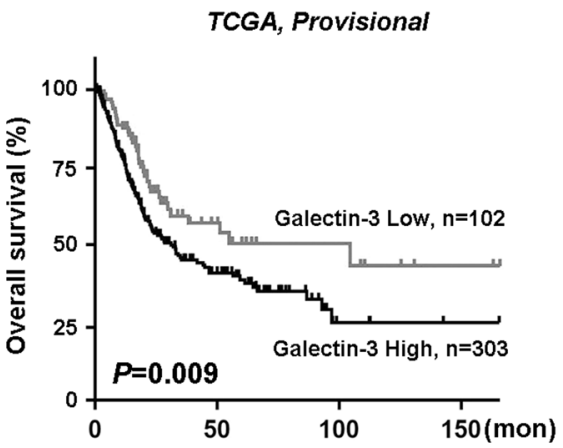

e

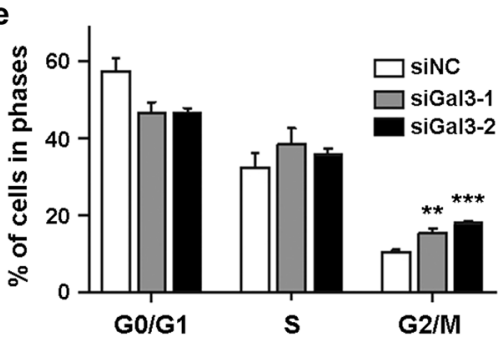

g

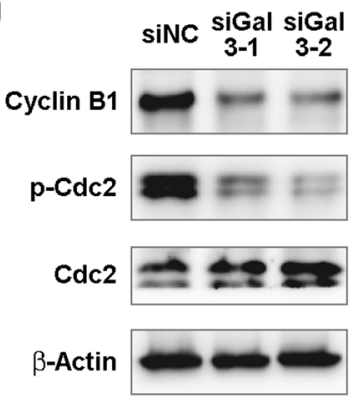

j

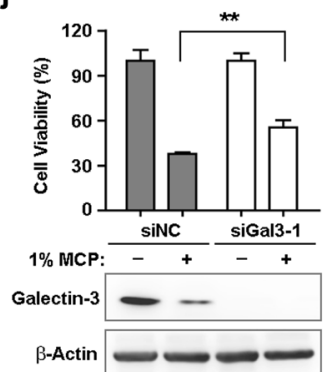

Fig. 5 Galectin-3 knockdown induced cell cycle arrest and apoptosis in T24 cells. a Comparison of normalized galectin-3 mRNA expression levels in patients without lymph node metastasis (N0, $n=73$ ) and with lymph node metastasis ( $\geq$ N1, $n=43$ ) in the TCGA Nature 2014 cohort. b Kaplan-Meier plots of cumulative overall survival (OS) were calculated for patients with low and high levels of galectin-3 expression in TCGA Nature 2014 cohort ( $n=129$, left panel) and TCGA provisional cohort $(n=405$, right panel). Lower quartile was determined as the cut-point. c Knockdown of galectin-3 by two different siRNAs (siGal3-1 and siGal3-2), using nontarget siRNA as a negative control (siNC). d Galectin-3 knockdown impaired the viability of UBC cells. e- $\mathbf{g}$ Galectin-3 knockdown induced $\mathrm{G}_{2} / \mathrm{M}$ cell cycle arrest in UBC cells. Quantification of cell cycle distribution (e), a representative cell cycle distribution pattern (f) and expression levels of $\mathrm{G}_{2} / \mathrm{M}$ phase transition regulators (g) were shown for the T24 cells with galectin-3 siRNA treatment. $\mathbf{h}$ Galectin-3 knockdown triggered Caspase-3 activities in UBC cells. $\mathbf{i}$ Effects of galectin-3 knockdown on apoptotic proteins and Akt signalings by Western blotting assay. $\mathbf{j}$ Galectin-3 knockdown partially rescued the inhibitory effect of MCP on cell viability in T24 cells. $48 \mathrm{~h}$ after siGal3-1 or siNC transfection, $1 \%$ MCP was applied to T24 cells for additional $48 \mathrm{~h}$. Cell viability was assessed using the SRB assay. Bars represented mean \pm SD. ${ }^{*} P<0.05 ;{ }^{* *} P<0.01 ;{ }^{* * *} P<0.001$

MCP inhibits the growth of UBC xenografts in vivo

To test whether MCP has inhibitory effect on tumor growth in vivo, T24 cells were injected subcutaneously in nude mice. When tumors became palpable, tumor-bearing animals were randomly divided into three groups ( $n=4$ for each group) for 6week treatment with low-dose (350 mg/kg MCP), high-dose (700 $\mathrm{mg} / \mathrm{kg} \mathrm{MCP)}$ and vehicle, respectively. The tumor growth was significantly decreased in both high-dose MCP group $(P<0.01)$ 

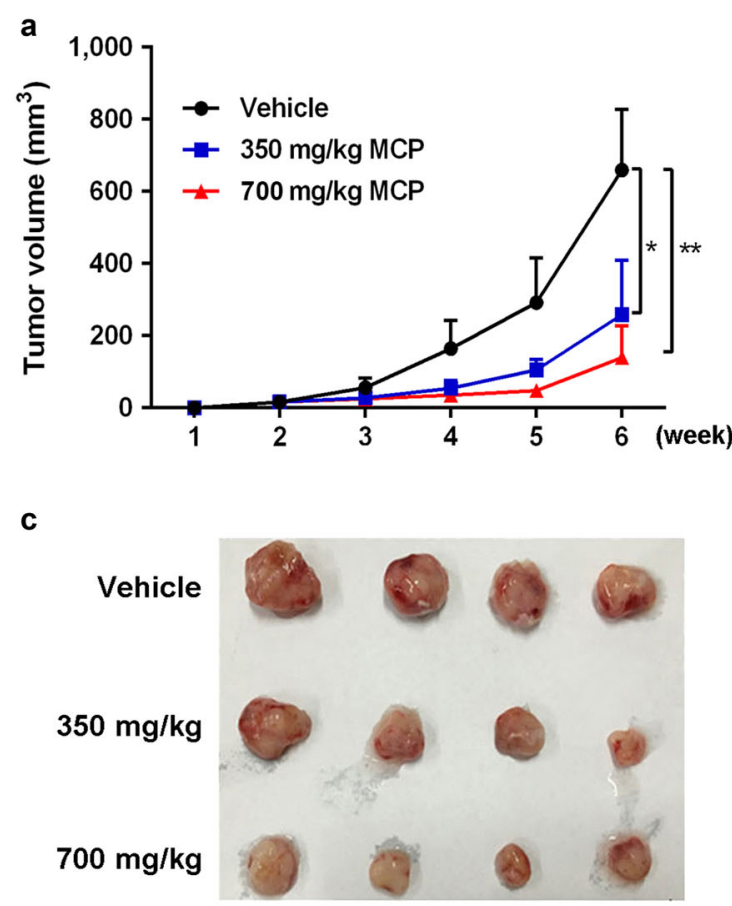

b

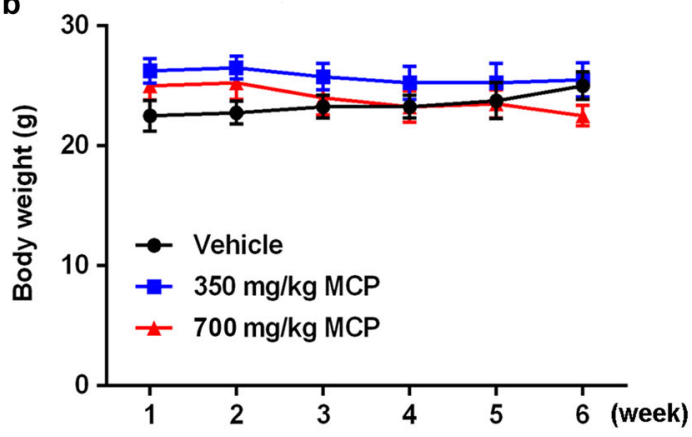

d

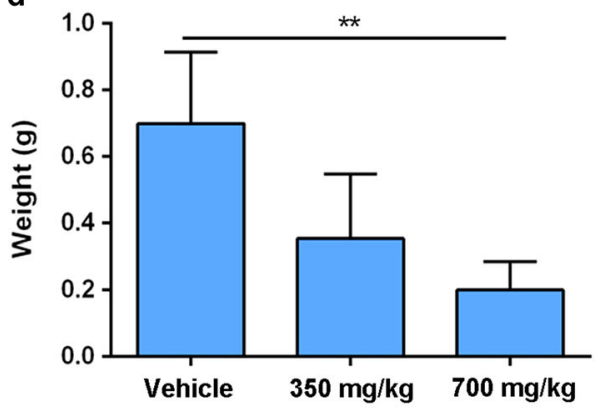

e

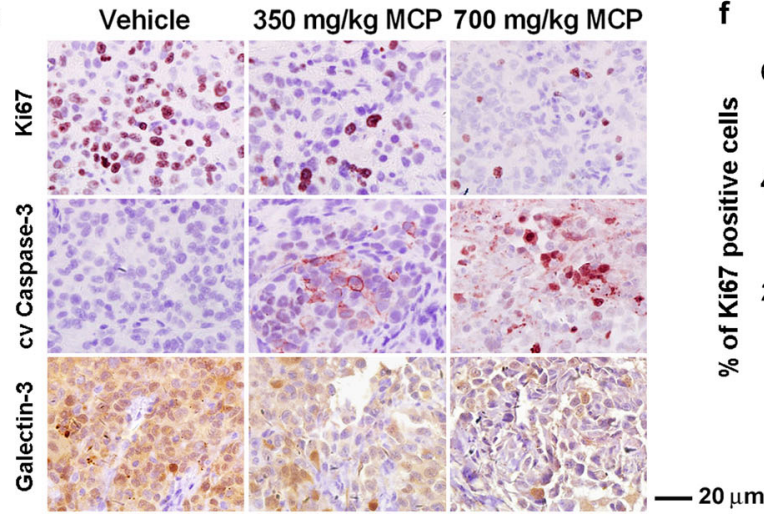

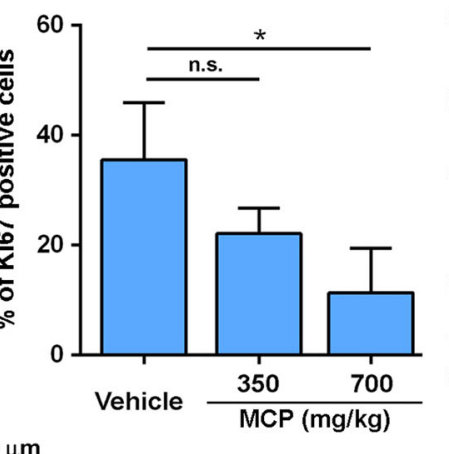

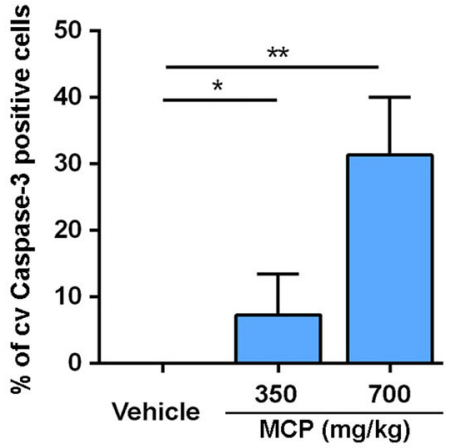

Fig. 6 MCP inhibited the growth of UBC xenografts in vivo. Tumor volume (a) and body weight (b) of T24 xenograft-bearing mice with MCP treatment for 6 weeks. Three experimental groups, including $700 \mathrm{mg} / \mathrm{kg} \mathrm{MCP,} 350 \mathrm{mg} / \mathrm{kg} \mathrm{MCP}$ and Vehicle control, were measured. Photograph (c) and the average weights (d) of T24 xenografts harvested at the endpoint. e Representative images of IHC staining for Ki67, cleaved Caspase-3 (cv Caspase-3) and Galectin-3 on tissue sections from MCP and vehicle-treated T24 xenografts. f Proliferation index and apoptosis index were quantified by the positive IHC staining for Ki67 and cleaved Caspase-3, respectively. Bars represented the mean \pm SD. n.s., $P \geq 0.05 ;{ }^{*} P<0.05 ;{ }^{*} P<0.01$

and low-dose MCP group $(P<0.05)$, compared with that in vehicle group (Fig. 6a). In addition, body weight of all animals did not show significant change under 6-week treatment of MCP (Fig. 6b). At the endpoint of this experiment, T24 xenografts were harvested (Fig. 6c) and weighted. Tumor weight of high-dose MCP group $(0.200 \pm 0.086 \mathrm{~g})$ was notably lighter than that of vehicle group $(0.669 \pm 0.215 \mathrm{~g} ; P<0.01$, Fig. $6 \mathrm{~d})$. Consistent with the data from in vitro assay, expression level of gelactin-3 protein was also decreased in MCP-treated T24 xenografts, comparing to the vehicle-treated animals (Fig. 6e). In addition, Ki67 (a marker for cell proliferation) and cleaved Caspase- 3 were examined by IHC staining in the T24 xenografts (Fig. 6e). Percentile of positive staining cells for Ki67 and cleaved Caspase-3 was quantified respectively (Fig. $6 \mathrm{f}$ ). High-dose MCP treatment led to a decline of the proliferative index by $68 \%(P<0.05)$ along with a remarkable increase of the apoptosis index $(P<0.01)$, compared with the vehicle group. The inhibitory effect on tumor growth by orally administered MCP $(700 \mathrm{mg} / \mathrm{kg})$ is demonstrated on UBC xenografts in vivo.

\section{DISCUSSION}

In this study, we first proved MCP, a kind of pectin originated from citrus, inhibited tumor growth via induction of cell cycle arrest and apoptosis in UBC cells in vitro and in vivo. MCP reduced the expression level of its potential binding protein galectin-3, which is overexpressed in high grade UBC samples and indicating poor overall survival. Subsequent downstream effect by MCP treatment includes the inactivation of Akt signaling pathway, but not MAPK signaling pathway.

As dietary fibers, pectins from different plants have been reported to possess cancer cell inhibitory effects [21]. For instance, apple pectin can induce apoptotic effects in MDA-MB-231 human breast cancer cells in vitro and 4T1 mouse breast cancer cells in vivo $[22,23]$. In addition, pectins originated from okra, sugar 
beet and Lonicera japonica were also demonstrated to have anticancer effects on melanoma, colon and pancreatic cancer cells, respectively [6, 24,25].

Modified by $\mathrm{pH}$ and heat treatment, MCP derived from citrus is one of the most studied pectin. Consistent with our findings in this study, $M C P$ can induce $G_{2} / M$ phase arrest in melanoma and prostatic JCA-1 cells $[7,26]$, as well as apoptosis in both mouse androgen-dependent and androgen-independent prostate cancer cells, with the cleavage of caspase-3 [8]. MCP could also suppress invasion or metastatic capacity of breast, prostate and colon cancer $[5,27,28]$. Besides, MCP can synergize with chemotherapeutic agent paclitaxel, cisplatin and doxorubicin on apoptosis induction in ovarian, prostate cancer cells and angiosarcoma cells, respectively [29-31]. In addition to its inhibitory effects on tumor cells, MCP enables functional activation of NK cells against cancer cells and inhibits capillary tube formation of HUVEC [28,32]. Overall, MCP has the tumor inhibitory effects on both tumor cells, including UBC cells demonstrated herein, and the tumor microenvironment cells.

MCP is a water-soluble citrus-derived polysaccharide fiber, which is rich in $\beta$-galactoside residues. The cytotoxic effect of MCP was shown through binding to the carbohydrate recognition domain of galectin-3 or downregulation galectin-3 [6, 33,34]. Galectin-3 is frequently overexpressed in a variety of neoplastic cell types and has been associated with cell proliferation, survival, cellular adhesion process and metastasis $[35,36]$. Based on the information of UBC patients from the public database TCGA, we found that MCP upregulation is correlated to high tumor grade with lymph node metastasis and may serve as a prognostic biomarker for UBC. We further demonstrated that targeting galetin- 3 by MCP in UBC cells led to $G_{2} / M$ cell cycle phase arrest and apoptosis, followed by the inactivation of Akt signaling pathway. Of note, knockdown of galectin-3 gene by RNA interference recapitulated the MCP-mediated inhibitory effects.

MCP has been reported to reduce MAPK signaling pathway in prostate cancer, which further induced Bim activity to apoptosis [8]. However, for this study, in T24 and J82 UBC cells with MCP treatment, the significant change of MAPK signaling pathway was undetectable. Instead, Akt signaling was abrogated by MCP in a dose-dependent manner, accompanied by reduced phosphorylation levels of Akt downstream proteins, such as Bad and S6RP. In another independent study, ectopic expression of galectin-3 conferred TRAIL resistance to J82 cells by elevating AKT activity [37]. These data suggest effector signaling pathways mediated by MCP are cell-context dependent and Akt signaling pathway may be a preference for MCP treatment in UBC cells.

In conclusion, our data showed that MCP suppressed UBC cell proliferation and survival in vitro and in vivo. As a natural dietary fiber, MCP is an attractive agent for chemoprevention and chemotherapeutics in UBC. The combined treatment of MCP and other therapeutic agents may be further investigated in future study.

\section{ACKNOWLEDGEMENTS}

This work was supported by the National Natural Science Foundation of China (81372168 to J. Y.), the Natural Science Foundation for Universities in Jiangsu Province (BK20151396 to J. Y.), One Hundred Talent Program of Chinese Academy of Sciences (to R. H.), and the Research Fund of Institutes for Drug Discovery and Development, Chinese Academy of Sciences (CASIMM0120164001 to R. H.). We thank the Institutional Technology Service Center of Shanghai Institute of Materia Medica for technical support.

\section{AUTHOR CONTRIBUTIONS}

J. Y., and R. H. designed research; T. F., D. L., H. N., D. L., J. S., X. H., and Y. D. performed research and analyzed data; T. F., D. L., H. N., J. Y., and R. H. wrote the paper; and M. G., S. Y., J. Y., and R. H. provided supervision.

\section{ADDITIONAL INFORMATION}

Competing interests: The authors declare no competing interests.

\section{REFERENCES}

1. Torre LA, Bray F, Siegel RL, Ferlay J, Lortet-Tieulent J, Jemal A. Global cancer statistics, 2012. Cancer J Clin. 2015;65:87-108.

2. Siegel RL, Miller KD, Jemal A. Cancer statistics, 2017. Cancer J Clin. 2017;67:7-30.

3. Vaidya A, Soloway MS, Hawke C, Tiguert R, Civantos F. De novo muscle invasive bladder cancer: is there a change in trend? J Urol. 2001;165:47-50.

4. Yeung $C$, Dinh $T$, Lee J. The health economics of bladder cancer: an updated review of the published literature. Pharmacoeconomics. 2014;32:1093-104.

5. Glinsky VV, Raz A. Modified citrus pectin anti-metastatic properties: one bullet, multiple targets. Carbohydr Res. 2009;344:1788-91.

6. Vayssade M, Sengkhamparn N, Verhoef R, Delaigue C, Goundiam O, Vigneron P, et al. Antiproliferative and proapoptotic actions of okra pectin on B16F10 melanoma cells. Phytother Res. 2010;24:982-9.

7. Jackson CL, Dreaden TM, Theobald LK, Tran NM, Beal TL, Eid M, et al. Pectin induces apoptosis in human prostate cancer cells: correlation of apoptotic function with pectin structure. Glycobiology. 2007;17:805-19.

8. Yan J, Katz A. PectaSol-C modifiedcitrus pectin induces apoptosis and inhibition of proliferation in humanand mouse androgen-dependent and- independent prostate cancer cells.Integr. Cancer Ther. 2010;9:197-203.

9. Gunning AP, Bongaerts RJ, Morris VJ. Recognition ofgalactan components of pectin by galectin-3. FASEB J. 2009;23:415-24

10. Ahmed H, AlSadek DM. Galectin-3 as a potential target to prevent cancer metastasis. Clin Med Insights Oncol. 2015;9:113-21.

11. Gao X, Liu J, Liu X, Li L, Zheng J. Cleavage and phosphorylation: important posttranslational modifications of galectin-3. Cancer Metastas. Rev. 2017;36:367-74.

12. Thijssen VL, Heusschen R, Caers J, Griffioen AW. Galectin expression in cancer diagnosis and prognosis: a systematic review. Biochim Biophys Acta. 2015;1855:235-47.

13. Ruvolo PP. Galectin 3 as a guardian of the tumor microenvironment. Biochim Biophys Acta. 2016;1863:427-37.

14. Zeinali M, Adelinik A, Papian S, Khorramdelazad $H$, Abedinzadeh M. Role of galectin-3 in the pathogenesis of bladder transitional cell carcinoma. Hum Immunol. 2015;76:770-4.

15. Canesin G, Gonzalez-Peramato P, Palou J, Urrutia M, Cordón-Cardo C, SánchezCarbayo M. Galectin-3 expression is associated with bladder cancer progression and clinical outcome. Tumour Biol. 2010;31:277-85.

16. Weinstein JN, Akbani R, Broom BM, Wang W, Verhaak RG, McConkey D, et al. Comprehensive molecular characterization of urothelial bladder carcinoma. Nature. 2014;507:315-22.

17. Abu-Elsaad NM, Elkashef WF. Modified citrus pectin stops progression of liver fibrosis by inhibiting galectin-3 and inducing apoptosis of stellate cells. Can J Physiol Pharmacol. 2016;94:554-62.

18. Zhang T, Zheng Y, Zhao D, Yan J, Sun C, Zhou Y, et al. Multiple approaches to assess pectin binding to galectin-3. Int J Biol Macromol. 2016;91:994-1001.

19. Nangia-Makker P, Conklin J, Hogan V, Raz A. Carbohydrate-binding proteins in cancer, and their ligands as therapeutic agents. Trends Mol Med. 2002;8:187-92.

20. Mori $Y$, et al. Binding of galectin-3, a $\beta$-Galactoside-binding lectin, to MUC1 protein enhances phosphorylation of extracellular signal-regulated kinase $1 / 2$ (ERK1/2) and akt, promoting tumor cell malignancy. J Biol Chem. 2015;290:26125-40.

21. Leclere L, Cutsem PV, Michiels C. Anti-cancer activities of $\mathrm{pH}$ - or heat-modified pectin. Front Pharmacol. 2013;4:128.

22. Delphi L, Sepehri H, Khorramizadeh MR, Mansoori F. Pectic-oligoshaccharides from apples induce apoptosis and cell cycle arrest in MDA-MB-231 cells, a model of human breast cancer. Asian Pac J Cancer Prev. 2015;16:5265-71.

23. Delphi L, Sepehri H. Apple pectin: a natural source for cancer suppression in $4 \mathrm{~T} 1$ breast cancer cells in vitro and express p53 in mouse bearing 4T1 cancer tumors, in vivo. Biomed Pharmacother. 2016;84:637-44.

24. Maxwell EG, Colquhoun IJ, Chau HK, Hotchkiss AT, Waldron KW, Morris VJ, et al. Modified sugar beet pectin induces apoptosis of colon cancer cells via an interaction with the neutral sugar side-chains. Carbohydr. Polym.. 2016;136:923-9.

25. Lin $L$, et al. Structural elucidation of a pectin from flowers of Lonicera japonica and its antipancreatic cancer activity. Int J Biol Macromol. 2016;88:130-7.

26. Hsieh TC, Wu JM. Changes in cell growth, cyclin/kinase, endogenous phosphoproteins and $\mathrm{nm} 23$ gene expression in human prostatic JCA-1 cells treated with modified citrus pectin. Biochem Mol Biol Int. 1995;37:833-41.

27. Liu HY, Huang ZL, Yang GH, Lu WQ, Yu NR. Inhibitory effect of modified citrus pectin on liver metastases in a mouse colon cancer model. World J Gastroenterol. 2008;14:7386-91. 
28. Nangia-Makker $P$, Hogan $V$, Honjo $Y$, Baccarini S, Tait $L$, Bresalier R, et al. Inhibition of human cancer cell growth and metastasis in nude mice by oral intake of modified citrus pectin. J Natl Cancer Inst. 2002;94:1854-62.

29. Wang Y, Nangia-Makker P, Balan V, Hogan V, Raz A. Calpain activation through galectin-3 inhibition sensitizes prostate cancer cells to cisplatin treatment. Cell Death Dis. 2010;1:e101.

30. Hossein G, Keshavarz M, Ahmadi S, Naderi N. Synergistic effects of PectaSol-C modified citrus pectin an inhibitor of Galectin-3 and paclitaxel on apoptosis of human SKOV-3 ovarian cancer cells. Asian Pac J Cancer Prev. 2013;14:7561-8.

31. Johnson KD, Glinskii OV, Mossine VV, Turk JR, Mawhinney TP, Anthony DC, et al. Galectin-3 as a potential therapeutic target in tumors arising from malignant endothelia. Neoplasia. 2007;9:662-70.

32. Ramachandran C, Wilk BJ, Hotchkiss A, Chau H, Eliaz I, Melnick SJ. Activation of human T-helper/inducer cell, T-cytotoxic cell, B-cell, and natural killer (NK)-cells and induction of natural killer cell activity against K562 chronic myeloid leukemia cells with modified citrus pectin. BMC Complement Altern Med. 2011;11:59.
33. Kolatsi-Joannou M, Price KL, Winyard PJ, Long DA. Modified citrus pectin reduces galectin-3 expression and disease severity in experimental acute kidney injury. PLoS One. 2011;6:e18683.

34. Menachem A, Bodner O, Pastor J, Raz A, Kloog Y. Inhibition of malignant thyroid carcinoma cell proliferation by Ras and galectin-3 inhibitors. Cell Death Discov. 2015;1:15047.

35. Cardoso AC, Andrade LN, Bustos SO, Chammas R. Galectin-3 determines tumor cell adaptive strategies in stressed tumor microenvironments. Front Oncol. 2016;6:127.

36. Wang L, Guo XL. Molecular regulation of galectin-3 expression and therapeutic implication in cancer progression. Biomed Pharmacother. 2016;78:165-71.

37. Oka N, Nakahara S, Takenaka Y, Fukumori T, Hogan V, Kanayama HO, et al. Galectin-3 inhibits tumor necrosis factor-related apoptosis-inducing ligandinduced apoptosis by activating Akt in human bladder carcinoma cells. Cancer Res. 2005;65:7546-53. 